\title{
Cirugía laparoscópica de la fístula vesico-vaginal: resultados de una experiencia multi-institucional*
}

\author{
Drs. OCTAVIO A. CASTILLO C. ${ }^{1,2}$, ANDRÉS HERNÁNDEZ P. ${ }^{3}$, MIGUEL GONZÁLEZ D. ${ }^{4}$, \\ GASTÓN LÓPEZ-FONTANA ${ }^{1}$, RICARDO YÁÑEZ M. ${ }^{1}$ \\ 1 Unidad de Urología, Clínica INDISA, Chile. \\ Facultad de Medicina, Universidad Andrés Bello, Chile. \\ CENTUR, Tijuana, México. \\ 4 Servicio de Urología, Hospital General Centro Médico Nacional La Raza e Instituto de Urología Avanzada. \\ Hospital Star Medica Lomas Verdes. México.
}

\begin{abstract}
\section{Laparoscopic surgery of vesicovaginal fistula: results of a multi institutional experience}

Introduction: Vesico-vaginal fistula is a rare complication of gynecologic surgery, with a high rate of surgical resolution O'Conor open technique. Aim: To report the results of a multi-institutional experience in laparoscopic repair of vesico-vaginal fistula. Material and Methods: Between January 2006 and June 2011, 21 laparoscopic vesico-vaginal fistula were performed. The surgical technique, demographic variables and results are described. Results: The mean age was 45.6 years. The average time between the diagnosis of the fistula and the laparoscopic repair was 15.23 months. The mean total operative time (bladder and laparoscopic) was 153.12 minutes and the average hospital stay was 2.7 days. The average time of bladder catheter was 9.4 days. There was a minimal recurrence of a fistula, repaired by a vaginal approach. Urethrocystography revealed indemnity of the repair in the other 20 cases. The overall success rate was $95.2 \%$ (20 out of 21 patients) Conclusions: The laparoscopic approach follows all principles for repair of a vesico-vaginal fistulas. It look like a good alternative in the hands of experienced surgeons.

Key words: Vesicovaginal fistula, hysterectomy, complications, laparoscopy, O'Conor.

\section{Resumen}

Introducción: La fístula vesico-vaginal es una complicación infrecuente de la cirugía ginecológica, con alta tasa de resolución quirúrgica con la técnica abierta tradicional de O'Conor. Objetivo: Comunicar los resultados de una experiencia multi-institucional en la reparación laparoscópica de las fístulas vesico-vaginales. Material y Método: Entre enero de 2006 y junio de 2011 se realizaron 21 reparaciones de fístulas vesicovaginales por vía laparoscópica. Se describe la técnica quirúrgica y se analizan las variables demográficas, quirúrgicas y resultados de la serie. Resultados: La edad media de las pacientes fue de 45,6 años. El tiempo promedio transcurrido entre el diagnóstico de la fistula y su reparación laparoscópica fue de 15,23 meses. El

*Recibido el 11 de enero de 2013 y aceptado para publicación el 11 de febrero de 2013.

Los autores no refieren conflictos de interés

Correspondencia: Dr. Octavio A. Castillo C. Avenida Santa María 1810, CP 7520440. Santiago, Chile. octavio.castillo@indisa.cl 
tiempo quirúrgico medio total (vesical y laparoscópico) fue de 153,12 min y el de hospitalización 2,7 días. El tiempo promedio de catéter uretro-vesical fue de 9,4 días. Hubo una mínima recidiva de una fístula, la cual fue reparada por vía vaginal. El control radiológico mediante cistografía reveló indemnidad de la reparación en el resto de los casos. La tasa global de éxito fue de 95,2\% (20 de 21 pacientes) Conclusiones: El abordaje laparoscópico permite cumplir con todos los principios para la reparación de las fístulas vesico-vaginales. La reducción de la morbilidad y la eficacia del procedimiento, lo transforman en una excelente alternativa en manos de cirujanos experimentados.

Palabras clave: Fístula vesico-vaginal, histerectomía, complicaciones, laparoscopia, O'Conor.

\section{Introducción}

La fístula vesico-vaginal (FVV) constituye una patología iatrogénica severa para la paciente. Su causa más frecuente en países desarrollados es la histerectomía ${ }^{1}$. Si bien su frecuencia es baja, con una incidencia estimada de 1 cada 1.800 histerectomías, una vez producida, constituye un problema social serio. El cierre espontáneo de la FVV mediante tratamiento conservador, colocando una sonda vesicouretral, es poco efectivo ( 7 al 12,5\%) ${ }^{2}$, por lo que el tratamiento quirúrgico cumple un rol fundamental en la corrección definitiva de esta patología.

Se han descrito numerosos procedimientos por vía transvaginal, vía transabdominal o abordaje combinado. La técnica quirúrgica por vía abdominal, con mejores resultados, ha sido la técnica de O'Conor, con una tasa de éxito entre $85 \%$ y $100 \%^{3}$, constituyendo actualmente la técnica de elección con la que se debe comparar cualquier otro procedimiento ${ }^{4}$. Nuestros resultados iniciales con la vía laparoscópica, en 8 pacientes, siguiendo los principios de la técnica clásica de $\mathrm{O}^{\prime}$ Conor, mostraron buenos resultados 5 . Debido a la baja frecuencia de las FVV en nuestro medio, y a la falta de series grandes de pacientes, decidimos realizar un estudio multi-institucional en 21 pacientes, en los cuales se empleó la misma técnica laparoscópica.

\section{Material y Métodos}

Entre enero de 2006 y junio de 2011 se realizaron 21 reparaciones por vía laparoscópica de una FVV. Todas las pacientes tenían el antecedente de una histerectomía de causa ginecológica. Se analizaron las siguientes variables: edad, tipo de cirugía ginecológica previa, tiempo entre el diagnóstico y la reparación, cirugía asociada, tiempo quirúrgico total (vesical y laparoscópico), complicaciones, tiempo de catéter vesical, tiempo de hospitalización y resultado de la reparación.

\section{Técnica quirúrgica}

Con la paciente en posición de litotomía modificada y mediante cistoscopia se cateterizan ambos uréteres con catéteres ureterales 5 Fr y se referencia la fístula utilizando un catéter ureteral de otro color (Figura 1). Se coloca un trocar de $10 \mathrm{~mm}$ a nivel umbilical para la óptica de 30 grados, un trocar de $10 \mathrm{~mm}$ pararectal izquierdo y 1 a 2 trocares de $5 \mathrm{~mm}$ pararectal y paraumbilical derechos (Figura 1).

Se realiza una cistotomía en sentido longitudinal siguiendo la técnica de O'Conor hasta localizar el catéter de la fístula y se secciona el tejido inflamatorio hasta obtener bordes de tejido normal. Se diseca el plano entre la vejiga y vagina, colocando un tampón vaginal para evitar el escape de $\mathrm{CO}_{2}$. La vagina es suturada en un plano transversal con sutura continua de Vicryl $\AA$ ) 2-0, mientras que la cistorrafia se lleva a cabo (Monocryl ${ }^{\circledR}$ ) 3-0, en un solo plano extramucoso continuo. Siempre se interpone epiplón entre ambos planos el cual se fija con un punto a la vagina. Se comprueba la hermeticidad de la sutura vesical llenando la vejiga con $200 \mathrm{ml} \mathrm{de}$ solución fisiológica. Se deja un drenaje aspirativo y una sonda vesical, la cual es retirada a los 10 días de la cirugía.

\section{Resultados}

La edad media de las pacientes fue de 45,6 años (32 a 67 años). En todos los casos la causa de la fís-



Figura 1. Aspecto de los catéteres colocados por vía cistoscópica para la identificación ureteral bilateral (color amarillo) y de la fístula (color verde). Se aprecia, además, la posición de los puertos laparoscópicos. 
tula fue una histerectomía de causa ginecológica, en tres de ellas se les había efectuado una ooforectomía en el mismo acto operatorio. El tiempo medio entre la histerectomía y la reparación laparoscópica fue de 15,3 meses ( 5 días a 156 meses). Una paciente consultó después de 13 años, tiempo durante el cual se manejó con pañales. El tiempo quirúrgico medio fue de 153,2 $\min$ ( 80 a $260 \mathrm{~min}$ ). En un caso fue necesario realizar un neoimplante ureteral, en el mismo acto quirúrgico, dada la proximidad del meato ureteral al borde de la fístula, con el riesgo de comprometerlo en la sutura. El tiempo medio de hospitalización fue de 2,7 días ( 1 a 10 días) y el tiempo promedio de sonda vesical fue de 9,4 días (5 a 17 días). No hubo complicaciones intraoperatorias. Hubo una recidiva de fístula a los 16 días de la cirugía, la cual se reparó por vía vaginal utilizando un colgajo de Martius ${ }^{6}$. El control radiológico mediante cistografía reveló indemnidad de la reparación en el resto de los casos. La tasa global de éxito primario en nuestra serie fue de un 95,2\% (20 de 21 pacientes).

\section{Discusión}

La FVV es una patología poco frecuente y sus consecuencias en la calidad de vida de las pacientes son devastadoras. En países desarrollados, la histerectomía abdominal es su principal causa $(85 \%)$ seguida de la radioterapia $(10 \%)^{7}$; mientras que en los países subdesarrollados el trabajo de parto prolongado es la causa más frecuente ${ }^{8}$.

El tratamiento de las FVV tiene cierto grado de controversia. Está descrito que el manejo conservador, mediante una sonda vesical mantenida por algunas semanas, tiene una tasa de cierre espontáneo sólo entre $7 \%$ y $12,5 \%{ }^{2,9}$. Esta baja probabilidad de éxito hace que el tratamiento quirúrgico desempeñe un rol sumamente importante en la corrección quirúrgica definitiva de esta patología. El abordaje transvaginal conlleva una menor morbilidad; sin embargo, las tasas de éxito no son superiores al $80 \%{ }^{8}$, siendo una técnica inadecuada para casos en los que la vagina es estrecha, hay radioterapia previa o cuando el útero está presente. En cuanto al abordaje abdominal clásico de $\mathrm{O}^{\prime}$ Conor, este se recomienda en casos de una difícil exposición del trayecto fistuloso ya sea tener una ubicación supratrigonal o cuando la vagina es estrecha, también cuando el trayecto fistuloso se encuentre muy cerca de algún orificio ureteral, ante presencia de múltiples fistulas o si se encuentra asociada a otras patologías pélvicas ${ }^{10}$. Independientemente de la técnica utilizada, todos los esfuerzos se deben dirigir a cerrar la FVV en el primer intento, lo que se logra en casi el $100 \%$ de los casos con la técnica transperitoneal de $\mathrm{O}^{\prime} \mathrm{Conor}$, la cual constituye el estándar de tratamiento quirúrgico actual. La vía laparoscópica permite reproducir fielmente la técnica del abordaje abdominal, aportando todas las ventajas de la cirugía mínimamente invasiva. Sin embargo, la necesidad de una sutura intracorpórea se presenta quizás como un importante obstáculo para desarrollar este abordaje. En nuestro caso, la experiencia en prostatectomía radical laparoscópica ha reducido nuestra curva de aprendizaje permitiendo incorporar el abordaje laparoscópico a nuestro arsenal terapéutico ${ }^{11}$. Recientemente publicamos nuestra experiencia en la reparación laparoscópica de FVV en 8 casos demostrando ser un abordaje factible y seguro que puede reproducir los pasos de la cirugía convencional ${ }^{5}$. No obstante, al ser una patología infrecuente, las series publicadas son

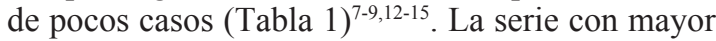

Tabla 1. Resumen de las series publicadas con más de 5 casos sobre reparación de fístulas vesico-vaginales por vía laparoscópica

\begin{tabular}{|c|c|c|c|c|c|c|}
\hline $\begin{array}{l}\text { Series } \\
\text { publicadas }\end{array}$ & $\begin{array}{c}\mathbf{n} \\
\operatorname{casos}\end{array}$ & $\begin{array}{c}\text { Estadía hospitalaria } \\
\text { (días) }\end{array}$ & $\begin{array}{l}\text { Tiempo de sonda } \\
\text { vesical (días) }\end{array}$ & Tasa complicaciones & $\begin{array}{c}\text { Tasa } \\
\text { conversión }\end{array}$ & $\begin{array}{c}\text { Tasa } \\
\text { de éxito }\end{array}$ \\
\hline Rizvi et al. ${ }^{7}$ & 8 & 1 & 14 & $0 \%$ & $0 \%$ & $100 \%$ \\
\hline Otsuka et al. ${ }^{9}$ & 7 & 7,2 & 28 & $\begin{array}{l}28,5 \%(2 / 7) \\
\text { - Infección urinaria } \\
\text { - Síndrome compartamental }\end{array}$ & $\begin{array}{c}14,2 \% \\
(1 / 7)\end{array}$ & $100 \%$ \\
\hline Sotelo et al. ${ }^{14}$ & 15 & 3 & 10,4 & $\begin{array}{l}13,3 \%(2 / 15) \\
\text { - Lesión intestinal } \\
\text { - Lesión arteria epigástrica }\end{array}$ & $0 \%$ & $93 \%$ \\
\hline Shah $^{15}$ & 25 & 4,5 & NR & $0 \%$ & $12 \%(3 / 25)$ & $86 \%$ \\
\hline Serie actual & 21 & 2,7 & 9,42 & $\begin{array}{l}4,76 \%(1 / 21) \\
\text { - Recidiva fístula }\end{array}$ & $0 \%$ & $95,2 \%$ \\
\hline
\end{tabular}

Los valores corresponden al promedio. NE: No especificado. 
número de casos fue publicada por Shah en el año $2009^{15}$. En su experiencia en 25 casos, comunica un tiempo medio operatorio de 145 min y una estadía hospitalaria de 4,5 días. Su tasa de conversión fue de $12 \%(3 / 25)$ y la tasa de éxito primario fue de $86 \%$ (19/22). Si bien nuestros resultados son de una serie multiinstitucional, la técnica quirúrgica fue la misma utilizada por 3 cirujanos (AH,MG,OAC), con una tasa global de éxito de $95,2 \%$ (20 de 21 pacientes). El caso de recidiva pudo ser resuelto fácil y precozmente por vía vaginal.

Recientemente se ha incorporado a nuestros recursos quirúrgicos la cirugía robótica (da Vinci ${ }^{\circledR}$ ) que es posible que pueda aportar ventajas comparativas o superiores a la vía laparoscópica ${ }^{16,17}$. Probablemente, en un futuro no muy lejano, las reflexiones respecto al tratamiento de las FVV estarán enfocadas a la utilidad de la robótica, quedando la laparoscopia relegada a un segundo término.

\section{Conclusiones}

La reparación laparoscópica de las fístulas vesicovaginales es un procedimiento seguro y eficaz, con resultados comparables con la cirugía convencional. Constituye una excelente alternativa para el manejo de esta patología, con todas las ventajas conocidas de la cirugía de mínima invasión.

\section{Referencias}

1. Miller EA, Webster GD. Current management of vesicovaginal fistulae. Curr Opin Urol. 2001;11:417-21.

2. Hilton P. Vesico-vaginal fistula: new perspectives. Curr Opin Obstet Gynecol. 2001;13:513-20.

3. O'Conor VJ Jr, Sokol JK, Bulkley GJ, Nanninga JB. Suprapubic closure of vesicovaginal fistula. J Urol. 1973;109:51-4.

4. Nesrallah LJ, Srougi M, Gittes RF. The O'Conor technique: the gold standard for supratrigonal vesicovaginal fistula repair. J Urol. 1999;161:566-8.
5. Miranda Utrera N, Foneron A, Castillo OA. Laparoscopic vesicovaginal fistula repair. Arch Esp Urol. 2012;65:887-90.

6. Rutman M, Rodríguez LV, Raz S. Vesicovaginal fistula: vaginal approach. En Female Urology. Third edition. Philadelphia. Editorial Sounders, 2008; pág.798.

7. Rizvi SJ, Gupta R, Patel S, Trivedi A, Trivedi P, Modi P. Modified laparoscopic abdominal vesico-vaginal fistula repair-"Mini-O'Conor" vesicotomy. J Laparoendosc Adv Surg Tech A. 2010;20:13-5.

8. Alonso y Gregorio S, Alvarez Maestro M, Cabrera Castillo PM, Hidalgo Togores L, de la Peña Barthel JJ. Laparoscopic repair of the vesicovaginal fistula (laparoscopic O’Conor repair). Actas Urol Esp. 2009;33:1133-7.

9. Otsuka RA, Amaro JL, Tanaka MT, Epacagnan E, Mendes JB Jr, Kawano PR, et al. Laparoscopic repair of vesicovaginal fistula. J Endourol. 2008;22:525-7.

10. Lee RA, Symmonds RE, Williams TJ. Current status of genitourinary fistula. Obstet Gynecol. 1988;72:313-9.

11. Castillo CO, Díaz CM, Vidal MI, Fonerón VA, Sánchez-Salas R, Vitagliano G. Prostatectomía radical laparoscópica extraperitoneal: Resultados de una curva de aprendizaje después de 310 casos. Rev Chil Cir. 2009;61:52-8.

12. Gözen AS, Teber D, Canda AE, Rassweiler J. Transperitoneal laparoscopic repair of iatrogenic vesicovaginal fistulas: Heilbronn experience and review of the literature. J Endourol. 2009;23:475-9.

13. Porpiglia F, Fiori C, Morra I, Ragni F, Vaccino D, Scarpa RM. Laparoscopic vesico-vaginal fistula repair: our experience and review of the literature. Surg Laparosc Endosc Percutan Tech. 2009;19:410-4.

14. Sotelo R, Mariano MB, García-Segui A, Dubois R, Spaliviero M, Keklikian W, et al. Laparoscopic repair of vesicovaginal fistula. J Urol. 2005;173:1615-8.

15. Shah SJ. Laparoscopic transabdominal transvesical vesicovaginal fistula repair. J Endourol 2009;23:1135-7.

16. Melamud O, Eichel L, Turbow B, Shanberg A. Laparoscopic vesicovaginal fistula repair with robotic reconstruction. Urology 2005;65:163-6.

17. Moy ML, Byun SY. Role of robotic surgery in urogynecologic surgery. Curr Opin Urol. 2010;20:70-4. 